\title{
A study on patient safety with reference to medication errors at a tertiary care hospital
}

\author{
Rajesh Dake*, I. Venkata Ramana
}

\begin{abstract}
${ }^{1}$ Department of Emergency Medicine, Emergency Physician, Apollo Hospitals, Kakinada, Andhra Pradesh, India ${ }^{2}$ Department of Operations, Apollo Hospitals, Kakinada, Andhra Pradesh, India
\end{abstract}

Received: 06 April 2020

Revised: 18 May 2020

Accepted: 22 May 2020

\section{*Correspondence:}

Dr. Rajesh Dake,

Email: drrajesh_d@apollohospitals.com

Copyright: (C) the author(s), publisher and licensee Medip Academy. This is an open-access article distributed under the terms of the Creative Commons Attribution Non-Commercial License, which permits unrestricted non-commercial use, distribution, and reproduction in any medium, provided the original work is properly cited.

\begin{abstract}
Background: The objective of this present study was to determine and evaluate the nature and types of medication errors (MEs) in a tertiary care hospital.

Methods: This was a prospective, observational study was conducted on medication errors in a tertiary care hospital, Kakinada during January 2019 to December 2019. MEs were categorized as prescription error (PE), transcription error (TE), dispensing error (DE), and administration error (AE). The case records and treatment charts were reviewed. The one-way ANOVA test for independent measures was done for statistical analysis.

Results: A total of 5792 patients were included during the study period. Total numbers of MEs were $\mathrm{n}=353(6.10 \%)$. The most common ME was TEs $n=191$ (54\%) followed by AEs $n=117$ (33\%). Nursing errors $n=316$ were more compared to doctors' errors $n=18$. The one-way Anova test for independent measures was done for statistical analysis; the f-ratio value is 6.44654 . The $\mathrm{p}$-value is 0.004332 . The result is significant at $\mathrm{p}<0.05$.

Conclusions: There is a need to establish effective ME reporting system to reduce its incidence and improve patient care and safety. Regular trainings and interdepartmental sharing of facts on medication errors should be done at regular intervals.
\end{abstract}

Keywords: Administration, Dispensing, Medication errors, Prescription, Transcription

\section{INTRODUCTION}

Patient safety is a fundamental practice for every healthcare provider. Medication safety is a major component in health care delivery system. Safe medication helps the patient to potentially scamper away from harm. The National coordinating council for medication error reporting and prevention (NCCMERP) has defined medication errors (MEs) as, "any preventable event that may cause or lead to inappropriate medication use or patient harm, while the medication is in the control of the health care professional, patient, or consumer". ${ }^{1}$ American Society of Hospital Pharmacists guidelines for MEs stated that "the incidence of MEs is not exactly known because of variations in different definitions of ME, different methods, or subject populations". 2 In India, the studies for the medication error rate in hospitalized patients have been documented to be as high as $25.7 \%$ and $15.34 \%$ in Uttarakhand and Karnataka respectively. ${ }^{3,4}$ Unfortunately, many cases of the ME remain undetected as there is no adverse effect on the patient.. If clinical significance or outcome does not adversely affect the patient, most of the MEs might have remained undetected. While some of the MEs also result into serious morbidity or mortality and have a considerable economic impact on the patient and health care system. The Institute of Medicine estimated the costs burdened due to medical errors in the US was approximately $\$ 37.6$ 
billion/year and about $\$ 17$ billion of it are associated with preventable errors. ${ }^{5}$ MEs increase morbidity, mortality, and economic burden to health care system. Medication error can lead to patient morbidity or mortality. ME can also cause emotional trauma to the patients. Medication errors include Lack of knowledge on medications and their actions, improper handling of computer software, incorrect medication, labelling and negligence when giving medicines that have been commonly detected.

In the study hospital, there were efforts taken to create awareness on medication safety. Few instances like the arrangement of medicines by colour coding, " $5 \mathrm{~S}$ management system". The trainings which include on handling look alike, sound alike medications, and high alert medications are the strategies implemented to prevent errors with medicines. Medication safety demand and role increase with the increase in use and growth of the pharmaceutical industries. This growth tends to increase in hazards, errors and adverse events which has trivial impact on patient outcomes and healthcare costs. ${ }^{6}$ Medications have become more complex with increasing in the number, variety of medications and different delivery routes for a single medication. Furthermore, human induced medication errors that include communication errors between nurses while changing their shift duties that lead to deliver of wrong medications to patients, Medication delivery errors by new nurses. Doctors and nurses have a major role in the use of medicine.

\section{METHODS}

This was a prospective, observational study was conducted on medication errors in a tertiary care hospital, Kakinada, Andhra Pradesh, India.

All the in-patient case records were reviewed during January 2019 to December 2019.

The case records and treatment charts were reviewed on daily basis from time of admission to till discharge. MEs were categorized as prescription error (PE), transcription error (TE), dispensing error (DE), and administration error (AE). MEs were also categorized according to healthcare providers.

\section{Inclusion criteria}

All the Inpatients who are reported to have medication errors were included.

\section{Exclusion criteria}

\section{All out patient drug prescriptions were excluded}

This study included the 5792 inpatient cases in this hospital. These are cases which were admitted during January 2019 to December 2019. Medication errors were analysed. The medication errors were categorised into different groups like administrative errors, nursing errors, doctors' errors, transcription errors, prescription errors, typing errors, dispensing errors. All the inpatient cases were segregated into the above listed medication errors.

Administrative errors include wrong time of administration, omission, wrong dose, wrong preparation and wrong administration rate. Transcription errors include incomplete prescriber orders, incomplete nurse hand writing and wrong use of abbreviations. Prescription errors include irrational, inappropriate, and ineffective, under dose and overdose prescribing. Typing errors include errors in data entry. Dispensing errors include pharmacist mistake in filling or dispensing the prescription

Nurse's errors include infections, dosage errors, wrong medicine to a person equipment's injury, communication errors with patient and families, limit overtime, documentation errors and negligence. Doctor's errors include anaesthesia, missed or delayed diagnosis, avoidable delay in treatment.

\section{Statistical analysis}

The percentage analysis was performed for all the medication errors to compare among different types of errors and among healthcare providers. The one-way ANOVA test was performed for errors to test the significance.

\section{RESULTS}

A total of 5792 Inpatients were included during the study period. Total numbers of medication errors were 353 $(6.10 \%)$ noted during study period which accounts for an average of 29.4 medication errors every month (Figure 1).

The most common medication error was transcription errors $n=191$ (54\%) (Figure 2 and 4) followed by administration errors $n=117(33 \%)$ (Figure 2 and 6) and prescription errors $n=45(13 \%)$ (Figure 2 and 5). Nursing errors $\mathrm{n}=316(89 \%)$ were more compared to doctors errors $n=18(5 \%)$ followed by typing errors $n=12(3.39 \%)$. The most common types of errors observed were improper dose (over dosage $(n=89)$ and less dose $(n=59)$, followed by wrong drug (drug other than the prescribed one, $(n=87)$, omission errors (failure to administer or failure to record the administration, $(n=82)$ and wrong time $(n=19)$, wrong rate (IV infusion rate was too fast, $(n=12)$. Medication errors are increased in the month of August $2019 \mathrm{n}=89(25.2 \%)$ of total medication errors. The F-ratio value was 6.44654 . The p-value is 0.004332 . The result was significant at $p<0.05$. Hence, the null hypothesis is rejected and alternate hypothesis is accepted. Percentage analysis was done to compare among different types of errors and among healthcare providers. 


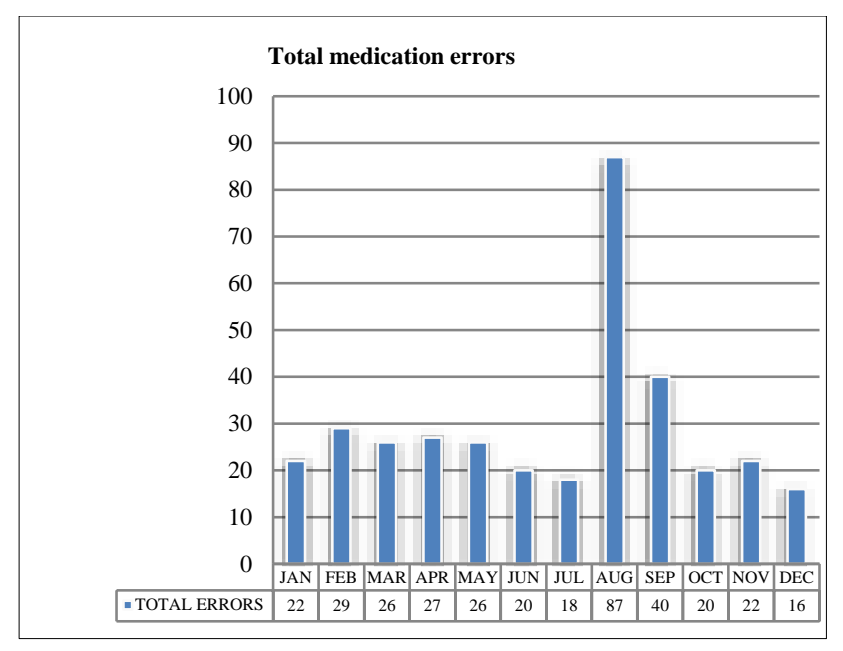

Figure 1: Monthly distribution of medication errors noted for the year 2019.

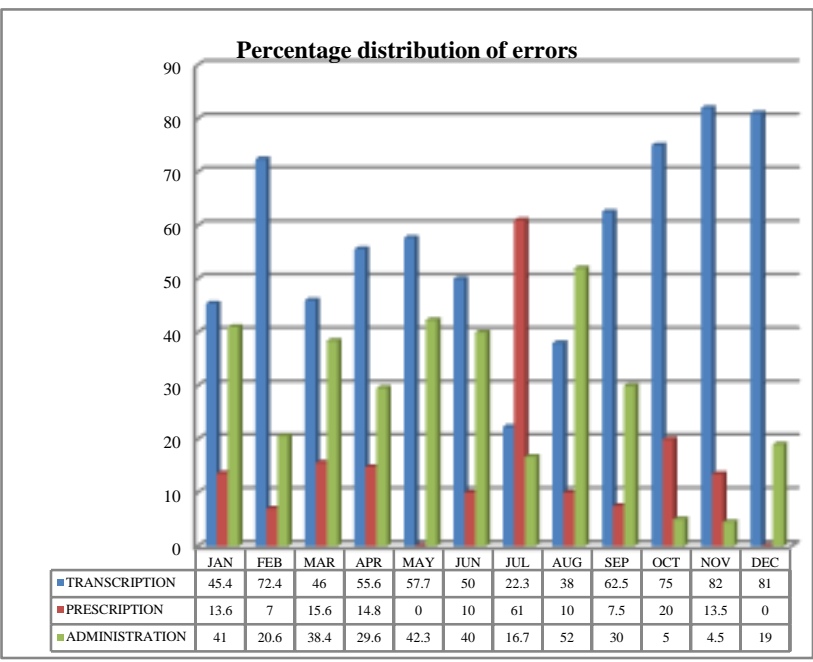

Figure 2: Monthly distribution of different types of medication errors noted for the year 2019.

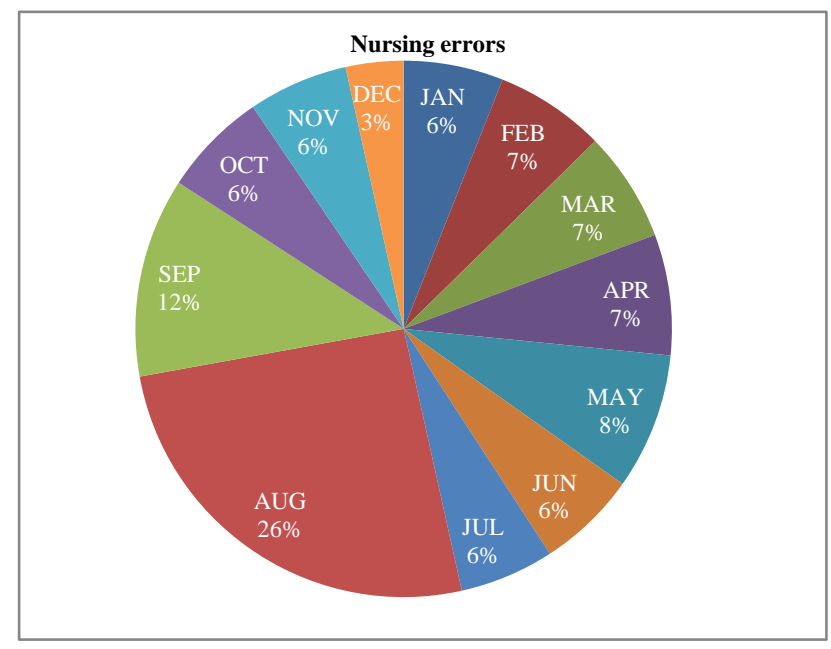

Figure 3: Monthly distribution of nursing related medication errors noted for the year 2019.

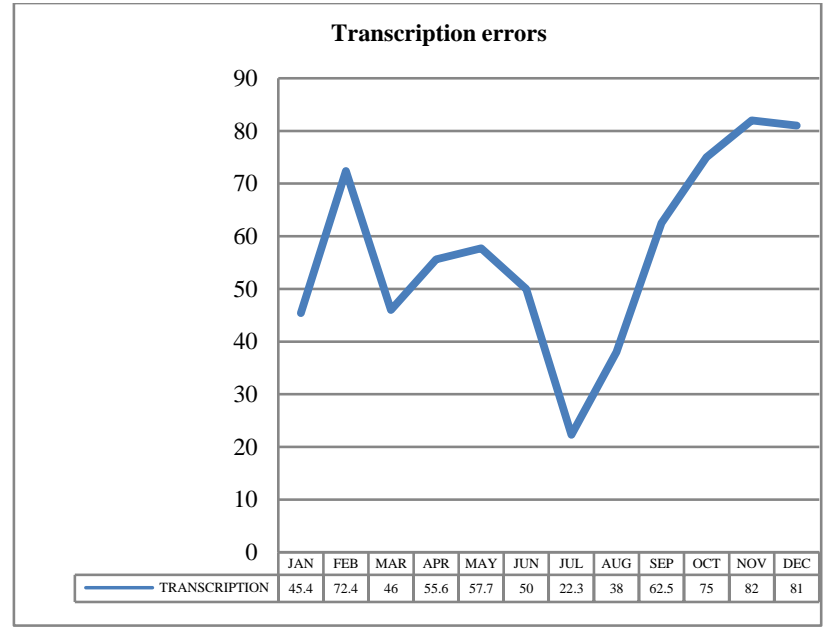

Figure 4: Monthly distribution of transcription medication errors noted for the year 2019.

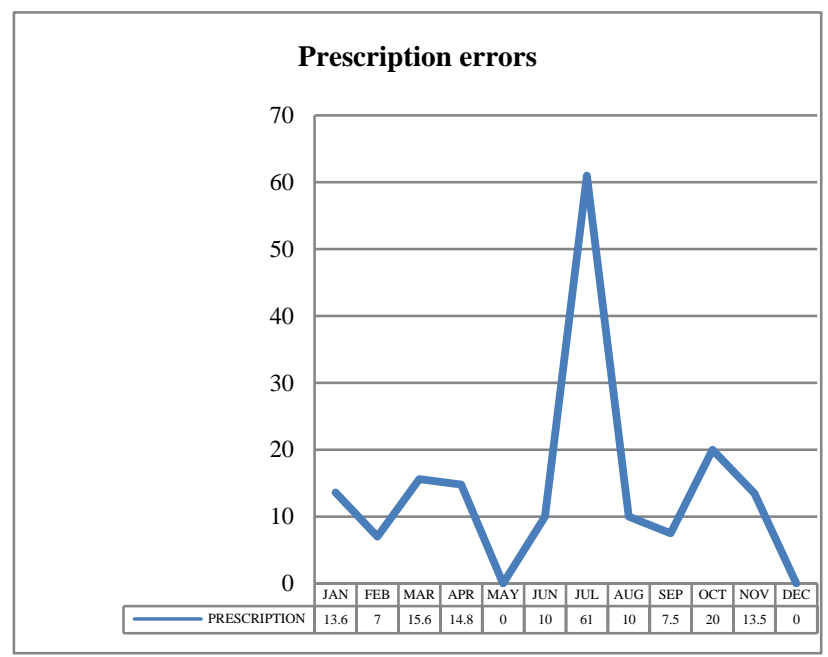

Figure 5: Monthly distribution of prescription medication errors noted for the year 2019.

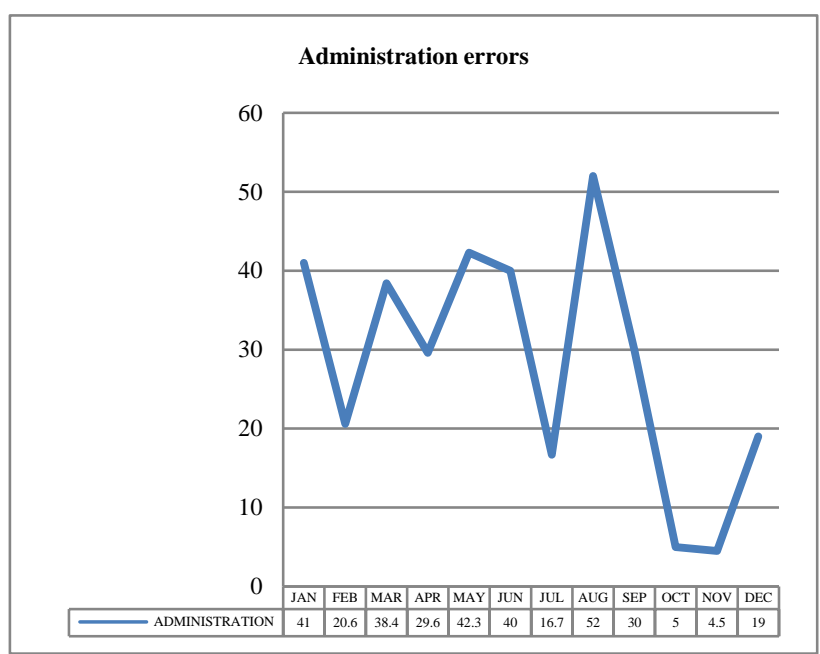

Figure 6: Monthly distribution of administration medication errors noted for the year 2019. 


\section{DISCUSSION}

Medication errors are a common cause for iatrogenic adverse events. They can lead to severe morbidity, prolonged hospitals stay, unnecessary diagnostic tests, unnecessary treatments and death. The multiple steps in the medication administration chain, from when a drug is prescribed to when a patient receives the drug, leads to significant scope for error. Most commonly, medication errors usually, occur at one of these points: ordering/prescribing, documenting, transcribing, dispensing, administering and monitoring. Medication error can lead to patient morbidity or mortality and even cause emotional trauma to patient. Lack of knowledge on medications and their actions, improper handling of computer software, incorrect medication, labelling and negligence when giving medicines are among the common mistakes that have been detected.

In present study, all the inpatients case sheets and their medication status were monitored, analysed on daily basis and arrived with following data. There are about 353 errors among total 5792 discharges which are about 6.10 $\%$. All the medication errors were further analysed according to their type of errors. Figure 2 depicts the types of errors and their distribution in each month during study period. Out of all the medication errors $(n=353)$ transcription errors $(n=191)$ are more compared to Administration errors $(n=117)$ and Prescription errors $(n=45)$. Nursing errors $(n=316)$ are more when compared to doctors' errors $(\mathrm{n}=18)$ and typing errors $(\mathrm{n}=12)$. Figure 6 depicts the percentage distribution of nursing errors in each month of the year 2019. This study observations are comparable to study done in Karnataka by Kumar et al. ${ }^{4}$ Monthly distribution of medication errors were analysed and were represented in a figure format as shown in Figure 1. Figure 1 depicts that there is abrupt increase in medication errors in the month of August, which might be due to increase in the number of new nurses and who were unaware of the process and protocols. In present study nursing errors $(n=316)$ are more when compared to doctors' errors $(n=18)$ and typing errors $(n=12)$. Figure 6 depicts the percentage distribution of nursing errors in each month of the year 2019.

According to the NCC MERP taxonomy, the medication administration error types were analysed. The most common types of errors observed were improper dose (over dosage $(n=89)$ and less dose $(n=59)$, followed by wrong drug (drug other than the prescribed one, $(n=87)$, omission errors (failure to administer or failure to record the administration, $(n=82)$ and wrong time $(n=19)$, wrong rate (IV infusion rate was too fast, $(n=12)$. This study showed that the most common medication AEs were inaccurate dose followed by inaccurate dosing interval. Studies done by Kumar et al, reported $17.4 \%$, while Agarwal and Joshi reported $45.5 \%$ dose errors. ${ }^{4,7}$ A study in Saudi Arabia showed $47.3 \%$ overdose errors. ${ }^{8}$ It has been documented in a study done by Parihar and Passi et al, that IV fluid administration is involved with wrong rates worldwide. ${ }^{9}$ The reason for inaccurate dose administration is due to lack of communication between health care professional team, missed labelling of IV fluid, and improper use of equipment's. An inaccurate dosing interval error was observed in this study which is higher than the reported by Kumar et al. ${ }^{4}$ Busy schedule, urge to complete work as early as possible, and missing double check/cross checking of prescription orders can lead to wrong route of administration. These reasons not only increase the patient risk, but also increase the cost of treatment.

In this study hospital, there were efforts taken to create awareness on medication safety. Few examples like the arrangement of medicines by colour coding and a " $5 \mathrm{~S}$ management system". There were also the trainings on Handling Look Alike, Sound Alike Medications, High alert medications which included the strategies to prevent errors with medicines. Both pharmacists and nurses each plays a pivotal role in this multi-disciplinary team to provide safety in handling medication, which extends the responsibility beyond supplying, preparing and administering medication as prescribed. For pharmacists, besides supplying medication to patients, also takes up the role in identifying drug-drug and drug-food interaction and educating patients in knowing their medication with the $5 \mathrm{R}$ (the right patient, the right drug, the right dose, the right route, and the right time) concept. Studies have shown that through patient education by pharmacist, internal and external ADE reports has decreased substantially and patients' adherence to medication improved. However, in relation with medication prescribed in ward, nurses, apart from clinical pharmacist are crucial in coordinating the care of patient with their clinical reasoning as they have the closest interaction with patients and doctors.

\section{CONCLUSION}

This study contributes in understanding of this much discussed worldwide theme, safety of the Patient. Through an analysis of notified medication errors which shows the occurrence of MEs at each phase of medication use cycle. There is a need to establish Effective ME reporting system to reduce its incidence and improve patient care and safety. Regular trainings and interdepartmental sharing of facts on Medication errors should be done at regular intervals. It is suggested that health institutions work towards a culture of safety, considering as a starting point, the planning of actions to promote the safety of the patient. To achieve this, it is necessary that all are involved, from managerial staff through to staff at all the various operational levels.

Funding: No funding sources Conflict of interest: None declared

Ethical approval: The study was approved by the Institutional Ethics Committee 


\section{REFERENCES}

1. Patel N, Desai M, Shah S, Patel P, Gandhi A. A study of medication errors in a tertiary care hospital. Perspective Clin Res. 2016;7(4):168.

2. ASHP guidelines on preventing medication errors in hospitals. Am J Hosp Pharm. 1993;50:305-14.

3. Gaur S, Sinha A, Srivastava B. Medication errors in medicine wards in a tertiary care teaching hospital of a hill state in India. Asian J Pharm Life Sci. 2012;2:56-63.

4. Kumar A, Venkateswarlu K, Ramesh A. A study of medication administration errors in a tertiary care hospital. Indian J Pharma Pract. 2011;4(2):37.

5. Kohn LT, Corrigan JM, Donaldson MS. To err is human: building a safer health system. Institute of medicine. Washington (DC): National Academies Press (US); 2000

6. Wei LY, Min TH, Ming EJC, Sheng JYB, Ahmad K. Qualitative research on medication safety among nurses and pharmacists in hospital Miri. Sarawak J Pharm. 2015;1:1-12.

7. Agarwal S, Joshi MC. A study of medication errors associated with prescription drug ordering. Rev Glob Med Healthc Res. 2011;2:166-72.

8. Ross LM, Wallace J, Paton JY. Medication errors in a paediatric teaching hospital in the UK: five years operational experience. Arch Dis Childhood. 2000;83(6):492-7.

9. Parihar M, Passi GR. Medical errors in pediatric practice. Indian Pediatr. 2008;45(7):586.

Cite this article as: Dake R, Ramana IV. A study on patient safety with reference to medication errors at a tertiary care hospital. Int J Basic Clin Pharmacol 2020;9:1015-9. 